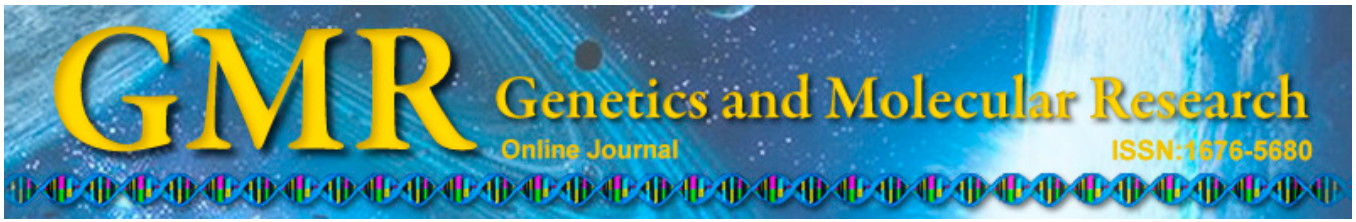

\title{
Genetic analysis of QTL for eye cross and eye diameter in common carp (Cyprinus carpio L.) using microsatellites and SNPs
}

\author{
S.B. Jin ${ }^{1,2}$, X.F. Zhang ${ }^{1}$, J.G. Lu ${ }^{1}$, H.T. Fu ${ }^{2}$, Z.Y. Jia ${ }^{1}$ and X.W. Sun ${ }^{1}$ \\ ${ }^{1}$ Heilongjiang River Fisheries Research Institute, \\ Chinese Academy of Fishery Sciences, Heilongjiang, Harbin, China \\ ${ }^{2}$ Key Laboratory of Freshwater Fisheries and Germplasm Resources Utilization, \\ Ministry of Agriculture, Freshwater Fisheries Research Center, \\ Chinese Academy of Fishery Sciences, Wuxi, China \\ Corresponding author: X.W. Sun \\ E-mail: sunxw2002@163.com
}

Genet. Mol. Res. 14 (2): 3557-3569 (2015)

Received March 25, 2014

Accepted October 29, 2014

Published April 17, 2015

DOI http://dx.doi.org/10.4238/2015.April.17.5

\begin{abstract}
A group of $107 \mathrm{~F}_{1}$ hybrid common carp was used to construct a linkage map using JoinMap 4.0. A total of 4877 microsatellite and single nucleotide polymorphism (SNP) markers isolated from a genomic library (978 microsatellite and 3899 SNP markers) were assigned to construct the genetic map, which comprised 50 linkage groups. The total length of the linkage map for the common carp was $4775.90 \mathrm{cM}$ with an average distance between markers of $0.98 \mathrm{cM}$. Ten quantitative trait loci (QTL) were associated with eye diameter, corresponding to $10.5-57.2 \%$ of the total phenotypic variation. Twenty QTL were related to eye cross, contributing to $10.8-36.9 \%$ of the total phenotypic variation. Two QTL for eye diameter and four QTL for eye cross each accounted for more than $20 \%$ of the total phenotypic variation and were considered to be major QTL. One growth factor related to eye diameter was observed on LG10 of the common carp genome, and three growth factors related to eye cross were observed on LG10,
\end{abstract}


LG35, and LG44 of the common carp genome. The significant positive relationship of eye cross and eye diameter with other commercial traits suggests that eye diameter and eye cross can be used to assist in indirect selection for many commercial traits, particularly body weight. Thus, the growth factor for eye cross may also contribute to the growth of body weight, implying that aggregate breeding could have multiple effects. These findings provide information for future genetic studies and breeding of common carp.

Key words: Mirror carp Cyprinus carpio L.; Quantitative trait loci; Eye cross and Eye diameter; Functional growth factor; Breeding; Correlation analysis

\section{INTRODUCTION}

The common carp (Cyprinus carpio L.) is the most extensively farmed fish species in China, and worldwide (Sun and Liang, 2004). Although productivity of this species has dramatically increased in recent decades, limited genetic research has been conducted on common carp breeding because of the low density of single nucleotide polymorphism (SNP) markers in linkage maps.

Genomic information can be used to advance genetic improvement programs in aquaculture, particularly the use of quantitative trait loci (QTL) mapping, which can lead to marker-assisted selection of desirable traits, as has already occurred in terrestrial livestock programs (Dekkers, 2004). However, genetic linkage maps are required to enable more detailed genetic studies. Such in-depth genetic studies may include the mapping of traits of commercial interest to a particular region of the genome, comparative genomics, physical cloning of mutants, and whole genome sequencing (Cnaani et al., 2004; Naruse et al., 2004). Fortunately, significant advances have been made in the construction of genetic linkage maps and the identification of QTL locations in the common carp in recent decades, including the construction of three genetic linkage maps using molecular markers (Sun and Liang, 2004; Zhang et al., 2010; Wang et al., 2011a) and the construction of comparative maps (Zheng et al., 2011). In addition, studies have also identified important commercial traits of the common carp on these maps in recent years. Of these studies, one conducted genetic analysis of the German mirror carp (Hou et al., 2007), others identified genes involved in the control of quantitative traits in common carp using QTL, such as cold tolerance (Sun and Liang, 2004) and muscle fiber density (Zhang et al., 2010), and others have conducted QTL analyses in other species (Zimmerman et al., 2005). Despite this progress, problems remain with previous studies. For example, some linkage maps were constructed using dominant markers (Zhang et al., 2010) or with a limited population, which makes it difficult to construct maps with greater levels of resolution (Sun and Liang, 2004).

SNPs are the most common type of variation in the genome, and they provide the best genome coverage for analyzing performance and production of traits. A genome with highdensity SNP coverage serves as a powerful tool for whole genome association studies because it can be used to detect linkage disequilibrium (Liu et al., 2011). Research has shown that SNP markers are very powerful markers for linkage mapping, construction of large-number populations, and identification of complex traits in humans (Wang et al., 1998), along with other 
model species (Zimdahl et al., 2004; Guryev et al., 2006). SNP markers have also been widely used to construct aquaculture fish linkage maps (Zheng et al., 2011; Lien et al., 2011; Wang et al., 2011b). The marker densities on these maps are believed to be relatively low because of insufficient markers. Consequently, genetic maps with low-density co-dominant marker coverage only provide small QTL regions of commercial traits, limiting functional gene analysis of the relatively large QTL regions. Recently, a number of microsatellites (Yue et al., 2004; Sun et al., 2005; Wang et al., 2007) have been identified as a practical tool for accurate construction of genetic linkage maps for large populations of common carp. Sufficient markers would enable QTL regions to be narrowed down and would increase the accuracy of common carp linkage maps. Such markers can also provide accurate QTL regions for commercial traits, including the detection of functional genes controlling the growth of different traits.

Eye diameter and eye cross are both important fish morphological characteristics and play essential roles in fish taxonomy (Yan et al., 2007; Liu et al., 2009). Furthermore, these traits are related to fish behavior, such as predation and predator avoidance (Kröger and Fernald, 1994; Ben-Simon et al., 2009). In a recent study, 445 polymorphic markers were used to identify the QTL location of head length, eye diameter, eye cross, body length, and body weight of carp on a carp linkage map, including 265 amplified fragment length polymorphism markers, 127 microsatellite markers, 37 EST-SSR markers, and 16 RAPD markers (Liu et al., 2009). In another study, 186 SSR and 321 SNP markers were used to carry out QTL location studies of eye diameter and eye cross in the common carp (Jin et al., 2012). Further in-depth investigations of the functional genes that control the characteristics of eye diameter and eye cross are required.

In this study, a high-density linkage map was constructed using common carp, and the genetic regions of eye diameter and eye cross were identified. In addition, the major functional regions of eye diameter and eye cross were individually identified by constructing a genetic linkage map using both microsatellite and SNP markers. The candidate functional genes responsible for the development of eye cross and eye diameter were determined from these major functional regions, significantly enhancing further breeding work.

\section{MATERIAL AND METHODS}

\section{Experimental fish}

German mirror common carp were obtained from the Songpu Aquaculture Experimental Station in Harbin, China. To obtain the largest genetic variation, males and females with relatively distant genetic descent were selected for breeding. Male and female individuals with the largest genetic variance were used to obtain the $\mathrm{F}_{1}$ generation. The mating of broodstock and production of the full-sib family were carried out at the Songpu Research Station (Harbin, China). Mature (3 years old), healthy broodfish in good condition were selected and stored at a hatchery in two tanks, with females and males kept separately. Eggs from a single female were fertilized with sperm from a single male and were spawned and hatched in a bowl. Water temperature during the incubation period was $22^{\circ} \mathrm{C}$. Following incubation, 107 embryos hatched and the fry were transferred to 107 rectangular tanks $(120 \mathrm{~L})$ with individual filtration circulation. Fish were fed every day and cultured under experimental conditions for six months. Eye cross and eye diameter were measured at six months post-hatching using Vernier calipers to an accuracy of $0.02 \mathrm{~mm}$, following the methods of Sun and Liang (2004), 
then the genotypes and phenotypes of the fish were determined. Eye diameter was measured as the inside diameter of the orbital, which is parallel to the head-to-tail axis and eye cross was measured as the straight-line distance between the edges of the fish orbitals.

\section{DNA extraction}

Fresh blood was extracted from each of the 107 individuals, and DNA was extracted from the fresh blood using a standard phenol-chloroform protocol (Sambrook and Russell, 2001). Genomic DNA was dissolved in double-distilled water, and the DNA quality was assessed on a $1 \%$ agarose gel. The DNA concentration was adjusted to $2.5 \mu \mathrm{g} / \mu \mathrm{L}$ based on the primary DNA concentration measured by spectrophotometer.

\section{Microsatellite and SNP genotyping}

One thousand microsatellite loci from BAC end sequences and 3000 microsatellite loci from genomic shotgun sequences, generated from the Roche 454 platform, were selected for marker development. A total of 1024 microsatellite loci were genotyped for the mapping population. Identification of SNP markers was conducted using the SLAF-seq method (Sun et al., 2013) and 4026 SNPs were identified for the population. JoinMap 4.0 was used to perform the linkage analysis. A total of 978 microsatellite markers and 3899 SNPs were amplified in the DNA samples. The total volume of the polymerase chain reaction was $15 \mu \mathrm{L}$, containing $1 \mathrm{U}$ Taq DNA polymerase, $1 \mathrm{X}$ PCR buffer $(10 \mathrm{mM}$ Tris- $\mathrm{HCl}, 50 \mathrm{mM} \mathrm{KCl}, 2.0 \mathrm{mM} \mathrm{MgCl}$, and $0.01 \%$ gelatin, $\mathrm{pH} 8.3$ ), $200 \mathrm{mM}$ dNTPs, $0.1 \mathrm{mM}$ of both the forward primer and reverse primer, and $100 \mathrm{ng}$ DNA templates. Thermal cycling was performed in a PCR System (Eppendorf Mastercycle pro S) in three steps. Cycling conditions consisted of an initial denaturation step at $94^{\circ} \mathrm{C}$ for $3 \mathrm{~min}$, followed by 25 cycles of denaturing at $94^{\circ} \mathrm{C}$ for $30 \mathrm{~s}$, annealing for $30 \mathrm{~s}$ (at a temperature specific for the primers being used), extension at $72^{\circ} \mathrm{C}$ for $30 \mathrm{~s}$, and a final extension step at $72^{\circ} \mathrm{C}$ for $5 \mathrm{~min}$. The amplified PCR products were visualized on an $8 \%$ polyacrylamide gel, using $1 \mathrm{X}$ TBE buffer, and the bands were analyzed using silver staining. The lengths of the PCR products were estimated using the Vision WorkLS software.

\section{Construction of linkage map and QTL location}

JoinMap 4.0 was used to construct the linkage map (Jacobs et al., 1995; Viruel et al., 1995) and MapQTL 4.0 was used to locate QTL using interval mapping (IM) methods (Van Ooijen, 2004). A limit of detection (LOD) score was set as the significant threshold for declaration of candidate QTL for both eye cross and eye diameter performed using IM. This threshold was set following a permutation test applied to each data set (1000 repetitions) $(\mathrm{P}=$ $0.05)$. QTL affecting these two traits were identified on a genome-wide scale, and the genomewide LOD significance threshold was 2.5.

\section{Detection of functional genes}

To identify genes contributing to the expression of eye diameter and eye cross traits, a blast search of QTL markers was performed against the draft genome of carp as a query and specific chromosome regions were extracted for gene prediction. Functional annotation of 
genes was performed using a BLASTX search (e-value $\left.<1 \mathrm{e}^{-5}\right)$ against the National Center for Biotechnology Information (NCBI) non-redundant protein database.

\section{Correlation analysis}

We measured the following traits six months after hatching: body weight, full length, body length, head length, eye diameter, eye cross, height, body thickness, caudal peduncle length, caudal peduncle height, and snout length. Microsoft Excel 2010 was used to perform the correlation analysis (Xie, 2010). When a population comprises more than 60 individuals, correlation values of $>0.193$ and $>0.254$ are considered to indicate correlation $(\mathrm{P}<0.05)$ and significant correlation $(\mathrm{P}<0.01)$ of variables, respectively (Zhou and Zheng, 1997). As such, these values were applied in this study.

\section{RESULTS}

\section{Measurements of eye diameter and eye cross}

The average difference is an index that indicates measurement accuracy, where the smaller the average difference, the more accurate the measurement is considered to be (Jin et al, 2012). Measurements of eye diameter ranged from $0.75-1.14 \mathrm{~cm}$ with an average $( \pm \mathrm{SD})$ of $0.9311 \pm 0.0077 \mathrm{~cm}$. The measurements of eye cross ranged from $1.69-2.70 \mathrm{~cm}$ with an average $( \pm \mathrm{SD})$ of $2.10 \pm 0.0205 \mathrm{~cm}$. These values indicate that the measurements could be used for further genetic analysis and QTL location research.

\section{Construction of the genetic map}

The linkage map containing 50 linkage groups was constructed using a total of 4877 markers ( 3899 of the 4026 SNP markers and 978 of the 1042 microsatellite markers). The total length of the inherited linkage map was $4775.90 \mathrm{cM}$. The map distance ranged from 47.498 cM on LG38 to $191.62 \mathrm{cM}$ on LG18, with an average distance between markers of $0.98 \mathrm{cM}$. The number of markers on each linkage group ranged from 32-166 with an average of 97.54 markers per group.

\section{QTL location}

Ten QTL were found to be responsible for the characteristics of eye diameter: qED1-5 (CA1602-SNP83693), qED2-5 (HLJ346-HLJ2013), qED3-5 (HLJ377-HLJ1909), qED1-21 (SNP49664-SNP67252), qED1-27 (HLJ3294-SNP27486), qED1-30 (SNP1629-SNP52392), qED1-44 (SNP32671-SNP4483), qED2-44 (SNP28085-SNP35232), qED1-45 (HLJ3407HLJ3396), and qED2-45 (SNP7445-SNP81574) (Table 1). Among these QTL linkage groups, the largest LOD value was held by the qED1-5 (CA1602-SNP83693) group. This group was responsible for $12.1 \%$ of the total phenotypic variation. The qED3-5 (HLJ377-HLJ1909) group had the smallest LOD value (2.55), explaining $10.5 \%$ of the total phenotypic variation. Of the total phenotypic variation, these ten linkage groups explained between $10.5 \%$ [group qED3-5 (HLJ377-HLJ1909)] and 57.2\% [group qED1-45 (HLJ3407-HLJ3396)] (Figure 1). The marker interval for eye diameter ranged from $1.786 \mathrm{cM}$ on LG 7 to $44.257 \mathrm{cM}$ on LG 42. 
Twenty QTL were found to control the expression of eye cross, including eleven QTL on linkage group 6. These QTL were located on the linkage groups qEC1-5 (SNP50851SNP79571), qEC2-5 (SNP8987-SNP3496), qEC1-6 (SNP6373-SNP9469), qEC2-6 (SNP59840-SNP14880), qEC3-6 (SNP26196-SNP25935), qEC4-6 (HLJ2556-SNP16225), qEC5-6 (SNP29602-SNP4802), qEC6-6 (SNP54288-SNP3418), qEC7-6 (SNP5961SNP1566), qEC8-6 (SNP3047-SNP3263), qEC9-6 (SNP20906-SNP29343), qEC10-6 (SNP53263-CA1598), qEC11-6 (SNP15767-HLJ853), qEC1-14 (SNP6081-HLJ2920), qEC133 (HLJE310-SNP14543), qEC2-33 (CA2183-HLJ1972), qEC3-33 (HLJ3143- SNP8212), qEC1-35 (SNP26965-CA1931), qEC1-39 (SNP29265-SNP16825), and qEC1-44 (SNP82796SNP71135) (Table 1). Among these QTL linkage groups, qEC1-6 (SNP6373-SNP9469) had the largest LOD value (4.09), explaining $24.8 \%$ of the total phenotypic variation. In contrast, qEC11-6 (SNP15767-HLJ853) had the smallest LOD value (2.53), explaining $12.8 \%$ of the phenotypic variation. The total phenotypic variation explained by these 20 linkage groups ranged from $10.8 \%$ by group qEC2-5 (SNP8987-SNP3496) to $36.9 \%$ by group qEC1-39 (SNP29265-SNP16825) (Figure 2). The marker interval for eye cross ranged from $0.581 \mathrm{cM}$ on LG 35 to $20.186 \mathrm{cM}$ on LG 32.
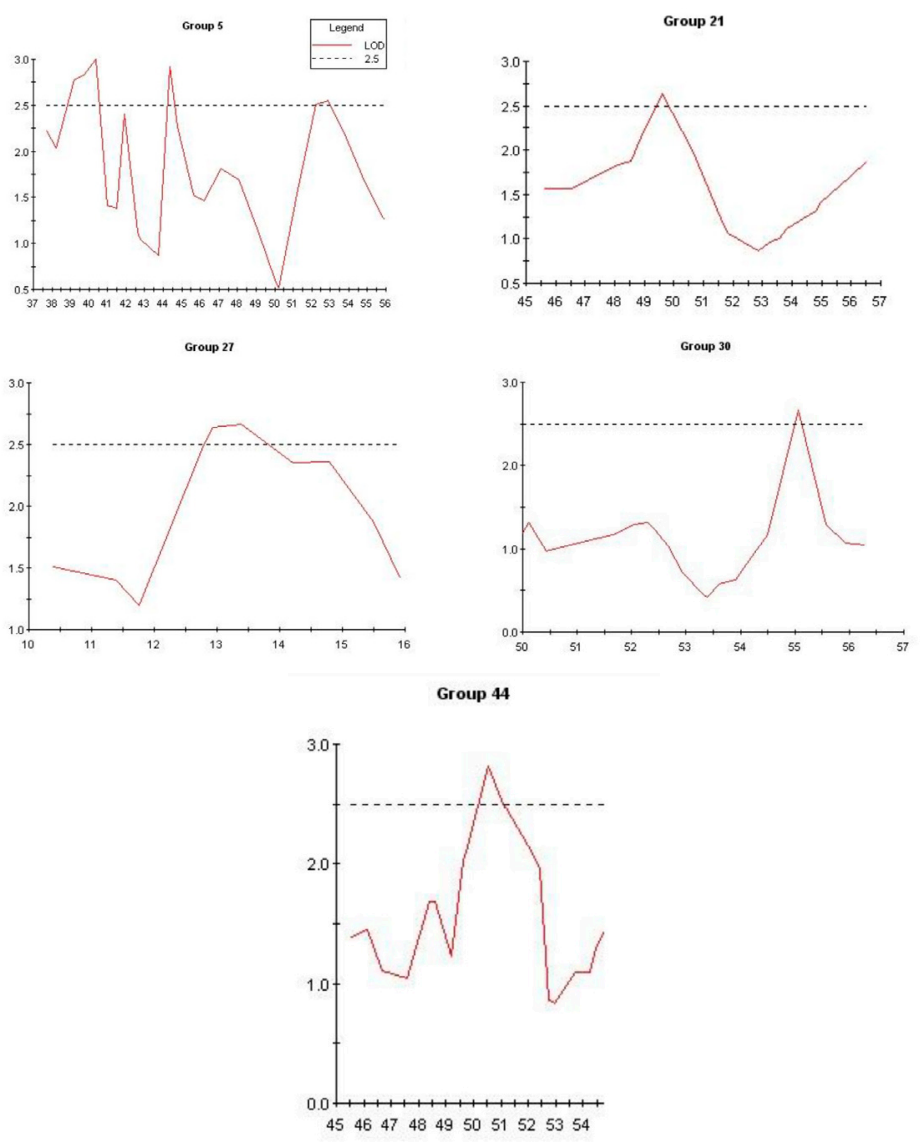

Figure 1. LOD curves for QTLs contributing to the eye diameter. The X-axis indicates marker distance and the Y-axis represents LOD with the dashed line indicating the threshold value of 2.5. 
Table 1. Eye diameter (ED) and eye cross (EC) QTLs and estimates of genetic effects.

\begin{tabular}{|c|c|c|c|c|c|c|}
\hline Linkage Group & QTL name & Marker interval & Marker interval (cM) & $\begin{array}{l}\text { Linkage group } \\
\text { size }(\mathrm{cM})\end{array}$ & LOD & $\begin{array}{c}\text { Variation } \\
(\%)\end{array}$ \\
\hline LG5 & qED1-5 & CA1602-SNP83693 & $39.8-41$ & 55.933 & 3 & 12.1 \\
\hline LG5 & qED2-5 & HLJ346-HLJ2013 & $43.7-44.8$ & 55.933 & 2.92 & 13.5 \\
\hline LG5 & qED3-5 & HLJ377-CA1909 & $50.2-55.9$ & 55.933 & 2.55 & 10.5 \\
\hline LG21 & qED-21 & SNP49664-SNP67252 & $48.9-51.8$ & 167.116 & 2.64 & 11.1 \\
\hline LG27 & qED1-27 & HLJ3294-SNP27486 & $12.9-14.2$ & 108.167 & 2.64 & 12.5 \\
\hline LG30 & qED1-30 & SNP1629-SNP52393 & $54.5-55.6$ & 124.087 & 2.67 & 11.4 \\
\hline LG44 & qED1-44 & SNP32671-SNP4483 & $49.8-51.1$ & 109.257 & 2.82 & 11.9 \\
\hline LG44 & qED2-44 & SNP28085-SNP35232 & 61.1-62.1 & 109.257 & 2.67 & 11.9 \\
\hline LG45 & $\mathrm{qED} 1-45$ & HLJ3407-HLJ3396 & $68.4-112.1$ & 126.423 & 2.85 & 57.2 \\
\hline LG45 & qED2-45 & SNP7445-SNP81574 & $61.2-63.1$ & 126.423 & 2.73 & 56.8 \\
\hline LG5 & $\mathrm{qEC} 1-5$ & SNP50851-SNP79571 & $10.3-11.8$ & 55.933 & 3.01 & 12.1 \\
\hline LG5 & $\mathrm{qEC} 2-5$ & SNP8987-SNP3496 & $14.4-15.1$ & 55.933 & 2.65 & 10.8 \\
\hline LG6 & qEC1-6 & SNP6373-SNP9469 & $11.7-14.4$ & 79.982 & 4.09 & 24.8 \\
\hline LG6 & $\mathrm{qEC} 2-6$ & SNP59840-SNP14880 & $31.1-31.8$ & 79.982 & 3.29 & 15.4 \\
\hline LG6 & qEC3-6 & SNP26196-SNP25935 & $29.5-30.4$ & 79.982 & 3.20 & 13.3 \\
\hline LG6 & qEC4-6 & HLJ2556-SNP16225 & $39.6-40.2$ & 79.982 & 3.18 & 15.2 \\
\hline LG6 & qEC5-6 & SNP29602-SNP4802 & $34.7-35.3$ & 79.982 & 3.08 & 15.2 \\
\hline LG6 & qEC6-6 & SNP54288-SNP3418 & $30.7-31.2$ & 79.982 & 2.94 & 12.1 \\
\hline LG6 & qEC 7-6 & SNP5961-SNP1566 & $37.2-37.4$ & 79.982 & 2.82 & 21.8 \\
\hline LG6 & qEC8-6 & SNP3047-SNP3263 & $36.5-36.8$ & 79.982 & 2.77 & 11.3 \\
\hline LG6 & qEC9-6 & SNP20906-SNP29343 & $32.2-32.4$ & 79.982 & 2.73 & 11.4 \\
\hline LG6 & qEC10-6 & SNP53263-CA1598 & $14.9-15.8$ & 79.982 & 2.60 & 13.4 \\
\hline LG6 & qEC11-6 & SNP15767-HLJ853 & $18.3-19.9$ & 79.982 & 2.53 & 12.8 \\
\hline LG14 & $\mathrm{qEC} 1-14$ & SNP6081-HLJ2920 & $35.4-37.7$ & 84.565 & 2.84 & 11.7 \\
\hline LG33 & qEC1-33 & HLJE310-SNP14543 & $64.6-65.6$ & 88.901 & 3.63 & 19.3 \\
\hline LG33 & $\mathrm{qEC} 2-33$ & CA2183-HLJ1972 & $51.0-51.5$ & 88.901 & 3.07 & 23.2 \\
\hline LG33 & $\mathrm{qEC} 3-33$ & HLJ3143-SNP8212 & $41.2-42.3$ & 88.901 & 2.64 & 13.1 \\
\hline LG35 & $\mathrm{qEC} 1-35$ & SNP26965-CA1931 & $52.9-53.6$ & 95.554 & 2.94 & 12.3 \\
\hline LG39 & qEC1-39 & SNP29265-SNP16825 & $0-5.2$ & 88.062 & 2.72 & 36.9 \\
\hline LG44 & $\mathrm{qEC} 1-44$ & SNP82796-SNP71135 & $32.7-37.0$ & 109.257 & 2.81 & 11.6 \\
\hline
\end{tabular}
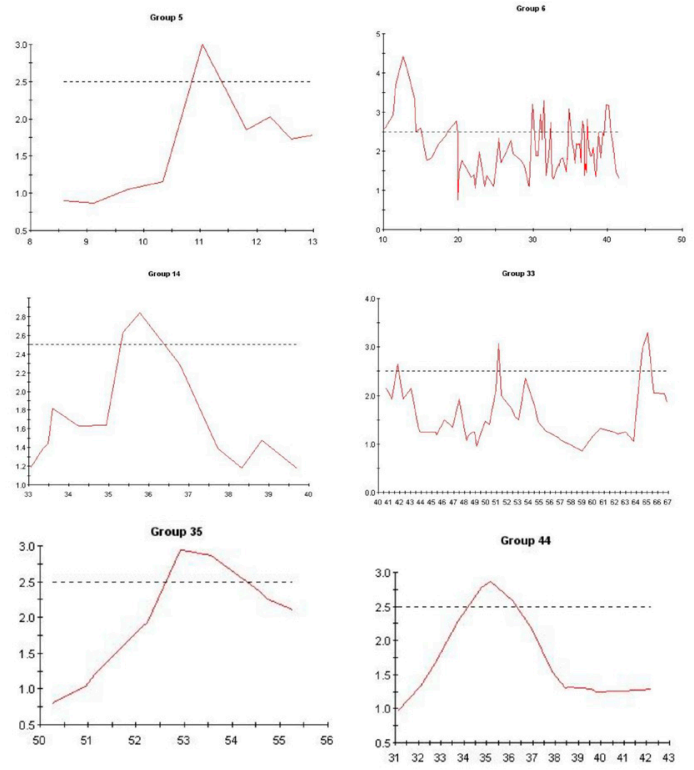

Figure 2. LOD curves for QTLs contributing to eye cross. The X-axis indicates marker distance and the Y-axis represents the LOD with the dashed line indicating the threshold value of 2.5. 


\section{Growth factor detection}

One growth gene was detected from the QTL qED5-3 with the function of growth arrestspecific 2 like 1. Three growth genes were detected from the QTL qEC5-2, qEC6-5, and qEC333 with the functions of putative growth hormone-regulated TBC protein, transforming growth factor-beta (TGF- $\beta$ ) family, and inhibitor of growth protein 5 , respectively. Their functions are detailed in Table 2 and Figure 3. These candidate genes were identified as being involved in the regulation of growth cycles, and they have previously been cited as being key candidate genes regulating growth rates (Lee et al., 1999; Moustakas and Heldin, 2008; Xing et al., 2011).

\begin{tabular}{|c|c|c|c|c|c|c|c|c|c|}
\hline \multirow{2}{*}{ QTL name } & \multirow{2}{*}{\multicolumn{2}{|c|}{$\begin{array}{l}\text { Associated Chr. } \\
\text { markers }\end{array}$}} & \multicolumn{2}{|c|}{ Marker location } & \multirow[t]{2}{*}{ Gene ID } & \multicolumn{2}{|c|}{ Gene location } & \multirow[t]{2}{*}{ Total length } & \multirow[t]{2}{*}{ Gene function } \\
\hline & & & From & To & & From & To & & \\
\hline qED-5-3 & CAFS1909 & 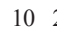 & 20321935 & 20321309 & 16558.gff.p. 0.0 & 19658977 & 19655410 & 22665327 & Growth arrest-specific 2 like 1 \\
\hline qEC-5-2 & SNP8987 & 10 & 5854802 & 5854492 & 430.gff.p.0.0 & 5854802 & 5854492 & 22665327 & $\begin{array}{l}\text { Growth hormone-regulated TBC } \\
\text { protein, putative }\end{array}$ \\
\hline qEC-6-5 & CAFS82 & 352 & 28025365 & 28024893 & 17348.gff.m.6.0 & 28013213 & 28011048 & 29126390 & $\begin{array}{l}\text { Transforming growth factor-beta } \\
\text { (TGF-beta) family }\end{array}$ \\
\hline qEC-33-3 & HLJE310 & 44 & 2767791 & 2768471 & 4563.gff.m.0.4 & 2126678 & 2126567 & 15901873 & Inhibitor of growth protein 5 \\
\hline
\end{tabular}
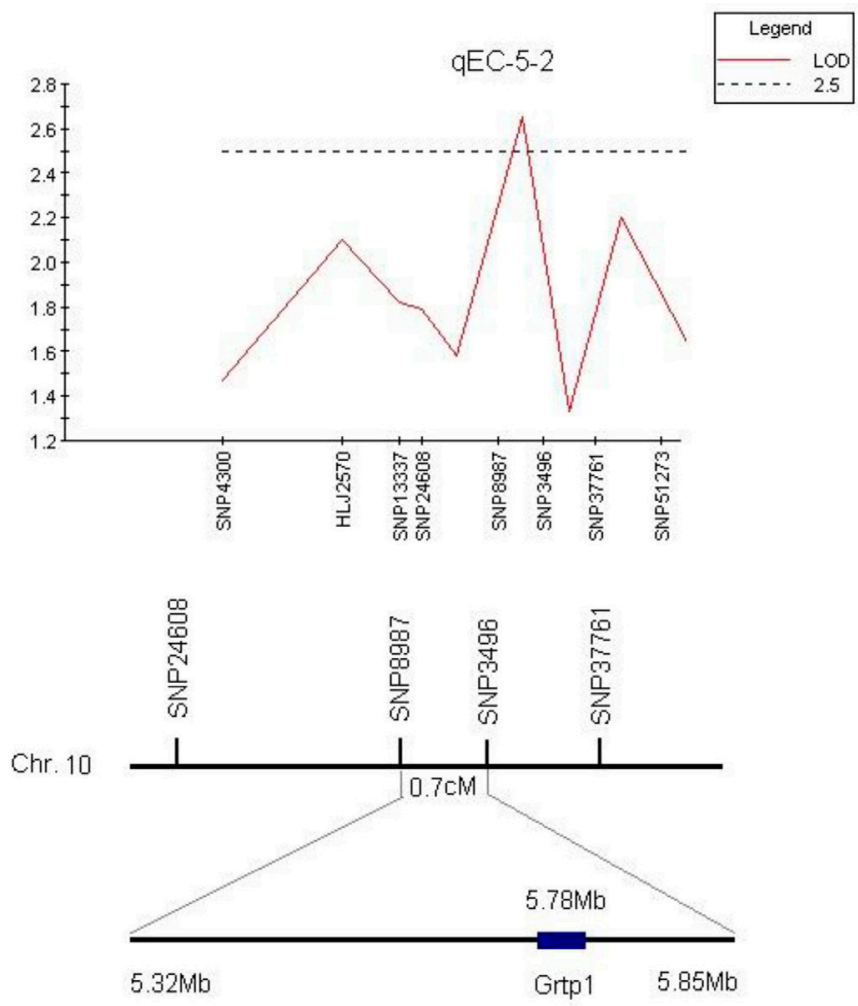

Figure 3. Candidate growth gene in the marker interval region of the carp genome. 


\section{Correlation analysis}

The results of the correlation analysis between eye diameter and eye cross and the other traits indicate that eye diameter and eye cross are positively correlated with all commercial traits $(\mathrm{P}<0.05$; Table 3$)$. Eye diameter and eye cross showed highly significant positive correlation with the important commercial traits $(\mathrm{P}<0.01)$, including body weight, full length, body length, height, and thickness. In particular, correlation was greater than 0.8 between eye cross and the following traits: body weight, full length, body length, height, and thickness.

\begin{tabular}{|c|c|c|c|c|c|c|c|c|c|c|c|}
\hline & $\begin{array}{l}\text { Body } \\
\text { weight }\end{array}$ & $\begin{array}{l}\text { Full } \\
\text { length }\end{array}$ & $\begin{array}{l}\text { Body } \\
\text { length }\end{array}$ & Height & Thickness & $\begin{array}{l}\text { Head } \\
\text { length }\end{array}$ & $\begin{array}{l}\text { Snout } \\
\text { length }\end{array}$ & $\begin{array}{c}\text { Eye } \\
\text { diameter }\end{array}$ & $\begin{array}{l}\text { Eye } \\
\text { cross }\end{array}$ & $\begin{array}{l}\text { Caudal } \\
\text { peduncle } \\
\text { length }\end{array}$ & $\begin{array}{c}\text { Caudal } \\
\text { peduncle } \\
\text { height }\end{array}$ \\
\hline Body weight & 1.0000 & & & & & & & & & & \\
\hline Full length & 0.9309 & 1.0000 & & & & & & & & & \\
\hline Body length & 0.9128 & 0.9900 & 1.0000 & & & & & & & & \\
\hline Height & 0.9716 & 0.8947 & 0.8706 & 1.0000 & & & & & & & \\
\hline Thickness & 0.8590 & 0.7318 & 0.7021 & 0.8578 & 1.0000 & & & & & & \\
\hline Head length & 0.7804 & 0.8092 & 0.7944 & 0.7540 & 0.6778 & 1.0000 & & & & & \\
\hline Snout length & 0.6148 & 0.5795 & 0.5796 & 0.5838 & 0.5321 & 0.4736 & 1.0000 & & & & \\
\hline Eye diameter & 0.5917 & 0.5498 & 0.5481 & 0.5527 & 0.4864 & 0.4069 & 0.5100 & 1.0000 & & & \\
\hline Eye cross & 0.8899 & 0.8470 & 0.8351 & 0.8633 & 0.7442 & 0.7853 & 0.6412 & 0.5661 & 1.0000 & & \\
\hline Caudal peduncle length & 0.4005 & 0.5745 & 0.5959 & 0.3712 & 0.2818 & 0.4007 & 0.0498 & 0.1935 & 0.3491 & 1.0000 & \\
\hline Caudal peduncle height & 0.8811 & 0.8330 & 0.8216 & 0.8725 & 0.7632 & 0.6747 & 0.5650 & 0.5276 & 0.7431 & 0.3750 & 1.0000 \\
\hline
\end{tabular}

\section{DISCUSSION}

This study aimed to identify the QTL regions for eye cross and eye diameter and to determine the candidate functional genes responsible for the development of these traits. A correlation analysis was performed to test whether the functional genes identified for eye diameter and eye cross potentially play an essential role for these characteristics and other important traits.

Recombination length was used to estimate the genome size of the common carp in this project and it was much larger than previously published for this species (Sun and Liang, 2004; Zhang et al., 2010; Wang et al., 2011b). The first linkage map for the common carp was constructed using dominant markers with a total recombination length of $4111 \mathrm{cM}$ based on 214 microsatellites and 54 RAPD markers (Sun and Liang, 2004). Further, additional codominant markers, including SNPs and microsatellites, were synthesized, making it possible to measure the genetic variation between different species and generations. Zhang et al. (2010) observed a genome length of $1852 \mathrm{cM}$ for a consensus map based on only 161 microsatellite markers. Wang et al. (2011b) obtained a total recombination length of $2805.85 \mathrm{cM}$ with an average linkage group length of $6.31 \mathrm{cM}$ based on 186 SSR markers and 321 SNP markers. In this study, the population size and number of markers were dramatically larger than those used in the previous studies. A total of 4877 polymorphic markers (3899 of 4026 SNP markers and 978 of 1042 microsatellite markers) were used to construct the genetic map with a population of 107. We obtained a total recombination length of $4777.09 \mathrm{cM}$ with an average linkage group length of $0.98 \mathrm{cM}$. Using plenty of markers can result in smaller average lengths between different markers. The linkage map comprised 50 linkage groups, which is consistent with the karyotype of the common carp (Yu et al., 1989), suggesting that sufficient markers 
were used to construct this high-quality linkage map. By using these results, the QTL of the common carp can be accurately pinpointed and can provide accurate information for future breeding programs.

Eye diameter and eye cross reported by Liu et al. (2009) and Jin et al. (2012) have been identified to be quantitative traits. According to previous studies, only two QTL were identified as being related to eye diameter and eye cross, explaining $5.62-9.77 \%$ and 8.29 $8.88 \%$ of the total phenotypic variation, respectively (Liu et al., 2009). Additionally, eight QTL were related to eye diameter and eleven QTL were related to eye cross, explaining 16.6036.70 and $13.60-31.60 \%$ of the total phenotypic variation, respectively. In the current study, ten QTL were related to eye diameter. Of the total variation, these linkage groups explained 10.5\% on LG5 qED3-5 (HLJ377-HLJ1909) and 57.2\% on qED1-45 (HLJ3407-HLJ3396). The QTL on LG45 explained the highest phenotypic variation (Table 1), thus, it is suggested as the main functional region for eye diameter. Twenty QTL were related to eye cross, and of the total phenotypic variation, these linkage groups explained 10.8\% on qEC2-5 (SNP8987SNP3496) to $36.9 \%$ on qEC1-39 (SNP29265-SNP16825). Eleven of these twenty QTL were located on LG6, implying that LG6 may be the main functional group controlling the expression of eye cross (Figure 2). Generally, the value of explained variation was closely related to trait variance within a family. Altogether, 30 QTL regions were found to be correlated to these two quantitative traits, with six regions (two regions for eye diameter and four regions for eye cross) considered to be significant because they each explained more than $20 \%$ of the total phenotypic variation. LG5 and LG44 were important for both eye diameter and eye cross because both QTL for eye diameter and eye cross were located on LG5 and LG44 simultaneously. In the present study, further QTL regions were identified for eye diameter and eye cross. Most importantly, these QTL regions are significantly smaller, and the accuracy of the genetic map is much higher, than reported in previous reports because many searches have been conducted in relation to the sequencing of the whole genome of common carp in recent years (Lien et al., 2011; Xu et al., 2011). Thus, sufficient SNP markers were available to construct the linkage map with a high-density of markers. As a consequence, the functional genes responsible for the growth and expression of eye cross and eye diameter were identified. Linkage groups 5 and 44 may contain functional genes that simultaneously control the growth of eye diameter and eye cross.

When the genome of a species is unknown, QTL may be useful for determining the putative functions of genes by observing their similarity to genes with known functions in other genomes through sequencing the identified region. It is often not the actual gene underlying the phenotypic trait, but rather a region of DNA that is closely linked with the gene (Jannink et al., 2001). This study is the first to identify functional genes responsible for the growth of commercial traits from QTL regions in common carp. In addition, a number of growth factors were observed that may regulate the growth of eye diameter and eye cross. Our results represent the first observations of major genetic growth factors and contribute toward understanding the regulation of eye diameter and eye cross growth in common carp.

In the present study, one growth factor (growth arrest-specific 2 like 1) was observed in the QTL region of eye diameter, and this growth factor is located on LG10 of the common carp genome. Three growth factors (growth hormone-regulated TBC protein, putative; TGF-beta family; inhibitor of growth protein 5) were found in the QTL regions of eye cross, and these growth factors are located on LG10, LG33 and LG45 of the common carp genome (Figure 3). Growth arrest-specific 2 like 1 is a member of the GAS2 family (Goriounov et 
al., 2003). GAS2 may play an important role in regulating chondrocyte proliferation and differentiation. This gene may also be involved in executing the apoptotic program in hindlimb interdigital tissues, acting as a death substrate for caspase enzymes (Lee et al., 1999). Growth hormone-regulated TBC protein (Grtp1) is a novel gene, and contains the TBC signature motif of GTPase activator proteins of Rab-like small GTPases. TGF- $\beta$ regulates cellular behavior in embryonic and adult tissues by binding to serine/threonine kinase receptors on the plasma membrane, which activates Smad molecules and additional signaling proteins that together regulate gene expression or cytoplasmic processes, such as cytoskeletal dynamics (Moustakas and Heldin, 2008). Inhibitor of growth protein 5 (ING5) is a new member of the ING family, identified by a computational homology search. Thus far, little research has been conducted on the function of ING5. ING5 can physically interact with p300 and p53 in vivo, and the over-expression of ING5 induces apoptosis in colorectal cancer cells. A study on mutation and downregulation of ING5 mRNA in oral squamous cell carcinoma suggests that this is a tumor suppressor gene (Xing et al., 2011). Correlation analysis can be used to examine the relationship between different commercial traits, and several studies have already proven that one commercial trait may assist the indirect selection for other commercial traits based upon their correlation relationship (Rasaei et al., 2011). In previous studies, eye cross and eye diameter were identified as being significantly correlated with body weight, and these two commercial traits share some QTL regions with body weight in the same generation of common carp (Jin et al., 2012). Based on correlation analysis, eye diameter and eye cross were found to be significantly positively correlated with many commercial traits, especially those with a higher positive correlation with body weight, which is consistent with the previous study (Jin et al., 2012). This implies that these two commercial traits may share QTL regions with body weight, and, thus, these two commercial traits may assist the indirect selection for body weight (Table 3; Jin et al., 2012).

Cluster phenomenon exists in many species and its ecological significance has been discussed because some QTL regions responsible for different traits have been observed clustered on the same QTL groups (Cai and Morishima, 2002). "Multifactorial linkages" may be the key factor for this cluster phenomenon followed by natural selection favoring co-adapted traits. Furthermore, pleiotropy based on a number of unknown key factor(s) controlling various traits through diverse metabolic pathways may be another possible reason for the clustering phenomenon (Cai and Morishima, 2002). Therefore, multiple traits could be improved simultaneously when closely linked markers are used to assist the breeding work based on close genetic characteristics, and aggregate breeding could achieve multiple effects. This indicates that the growth factors responsible for the regulation of eye diameter and eye cross in this study may also simultaneously control the growth of body weight. Therefore, studies on the growth factors observed here may also advance improvements in body weight, enhancing the commercial value of the carp population. These findings provide useful information for genetic studies and will benefit future breeding programs for common carp.

In conclusion, this study has accurately located eye diameter and eye cross on the linkage map of common carp. A number of growth factors related to the growth of eye diameter and eye cross have been identified, which has valuable implications for aquaculture breeding programs. This research also showed that eye diameter and eye cross may be used for indirect selection for important commercial traits and aggregate breeding could achieve multiple effects. 


\section{ACKNOWLEDGMENTS}

The authors are grateful to the Liwen English editing company for help in improving the English language. Research supported by a grant from the National High Technology Research and Development Program of China ("863" Program; \#2011AA100402), a key grant of Chinese National Programs for Fundamental Research and Development (\#2010CB126305), and a special research grant for non-profit public service (\#2009HSYZX-SJ-07).

\section{REFERENCES}

Ben-Simon A, Ben-Shahar O and Segev R (2009). Measuring and tracking eye movements of a behaving archer fish by real-time stereo vision. J. Neurosci. Methods. 184: 235-243.

Cai W and Morishima H (2002). QTL clusters reflect character associations in wild and cultivated rice. Theor. Appl. Genet. 104: 1217-1228.

Cnaani A, Zilberman N, Tinman S, Hulata G, et al. (2004). Genome-scan analysis for quantitative trait loci in an F 2 tilapia hybrid. Mol. Genet. Genomics 272: 162-172

Dekkers JC (2004). Commercial application of marker-and gene-assisted selection in livestock: strategies and lessons. $J$. Anim. Sci. 82: E313-E328.

Goriounov D, Leung CL and Liem RK (2003). Protein products of human Gas2-related genes on chromosomes 17 and 22 (hGAR17 and hGAR22) associate with both microfilaments and microtubules. J. Cell Sci. 116: 1045-1058.

Guryev V, Koudijs MJ, Berezikov E, Johnson SL, et al. (2006). Genetic variation in the zebrafish. Genome Res. 16:491-497.

Hou N, Zhang Y, Lu CY, Li Y, et al. (2007). Genetic potential analysis of Germany mirror carp (Cyprinus carpio L.) using microsatellite markers. Hereditas 29: 1509-1518.

Jacobs JM, Van Eck HJ, Arens P, Verkerk-Bakker B, et al. (1995). A genetic map of potato (Solanum tuberosum) integrating molecular markers, including transposons, and classical markers. Theor. Appl. Genet. 91: 289-300.

Jannink J, Bink MC and Jansen RC (2001). Using complex plant pedigrees to map valuable genes. Trends Plant Sci. 6: 337-342.

Jin S, Zhang X, Jia Z, Fu H, et al. (2012). Genetic linkage mapping and genetic analysis of QTL related to eye cross and eye diameter in common carp (Cyprinus carpio L.) using microsatellites and SNPs. Aquaculture 358-359: 176-182.

Kröger RH and Fernald RD (1994). Regulation of eye growth in the African cichlid fish Haplochromis burtoni. Vision Res. 34: 1807-1814.

Lee KKH, Tang MK, Yew DT, Chow PH, et al. (1999). Gas2 is a multifunctional gene involved in the regulation of apoptosis and chondrogenesis in the developing mouse limb. Dev. Biol. 207: 14-25.

Lien S, Gidskehaug L, Moen T, Hayes BJ, et al. (2011). A dense SNP-based linkage map for Atlantic salmon (Salmo salar) reveals extended chromosome homeologies and striking differences in sex-specific recombination patterns. BMC Genomics 12: 615.

Liu JH, Zhang Y, Chang YM, Liang LQ, et al. (2009). Mapping QTLs related to head length, eye diameter and eye cross of common carp (Cyprinus carpio L.). Hereditas 31: 508-514.

Liu S, Zhou Z, Lu J, Sun F, et al. (2011). Generation of genome-scale gene-associated SNPs in catfish for the construction of a high-density SNP array. BMC Genomics 12:53.

Moustakas A and Heldin C (2008). Dynamic control of TGF- $\beta$ signaling and its links to the cytoskeleton. FEBS Lett. 14: 2051-2065.

Naruse K, Tanaka M, Mita K, Shima A, et al. (2004). A medaka gene map: the trace of ancestral vertebrate protochromosomes revealed by comparative gene mapping. Genome Res. 14: 820-828

Rasaei A, Ghobadi ME, Ghobadi M and Abdi-niya K (2011). The study of traits correlation and path analysis of the grain yield of the peas in semi-dry conditions in Kermanshah. Proceedings of the International Conference on Food Engineering and Biotechnology 9: 246-249.

Sambrook J and Russell D (2001). Molecular Cloning: A Laboratory Manual, 3rd edn. Cold Spring Harbor Press, Cold Spring Harbor, NY.

Sun X and Liang L (2004). A genetic linkage map of common carp (Cyprinus carpio L.) and mapping of a locus associated with cold tolerance. Aquaculture 238: 165-172.

Sun X, Jia Z, Wei D, Lu C, et al. (2005). Comparison between magnetic beads enriched and small inserted fragment library for microsatellite sequences of common carp. J. Fish. China 12: 126-132. 
Sun X, Liu D, Zhang X, Li W, et al. (2013). SLAF-seq: An efficient method of large-scale de novo SNP discovery and genotyping using high-throughput sequencing. PLoS One 8: e58700.

Van Ooijen JW (2004). MAPQTL ${ }^{\circledR}$ 5, Software For The Mapping of Quantitative Trait Loci in Experimental Populations. Kyazma B. V., Wageningen.

Viruel MA, Messeguer R, de Vicente MC, Garcia-Mas J, et al. (1995). A linkage map with RFLP and isozyme markers for almond. Theor. Appl. Genet. 91: 964-971.

Wang DG, Fan JB, Siao CJ, Berno A, et al. (1998). Large-scale identification, mapping, and genotyping of singlenucleotide polymorphisms in the human genome. Science 280:1077-1082.

Wang D, Liao X, Cheng L, Yu X, et al. (2007). Development of novel EST-SSR markers in common carp by data mining from public EST sequences. Aquaculture 271: 558-574.

Wang XP, Zhang XF, Li WQ, Zhang TQ, et al. (2011a). Analysis of feed conversion ratio, bodyweight, body thickness traits of common carp (Cyprinus carpio L.) using SSR, EST-SSR, SNP markers. J. Fishery Sci. China 18: 565-573.

Wang XP, Sun XW, Li WQ, Zhang TQ, et al. (2011b). The construction of genetic linkage map of common carp (Cyprinus carpio L.). J. Shanghai Ocean Univ. 20: 641-648.

Xie Q (2010). Practical Tutorial of Excel 2007 spreadsheets. Tsinghua Univ. Press, China.

Xing YN, Yang X, Xu XY, Zheng Y, et al. (2011). The altered expression of ING5 protein is involved in gastric carcinogenesis and subsequent progression. Hum. Path. 42: 25-35

Xu P, Li J, Li Y, Cui R, et al. (2011). Genomic insight into the common carp (Cyprinus carpio) genome by sequencing analysis of BAC-end sequences. BMC Genomics 12:188.

Yan X, Liang L, Sun X and Cao D (2007). Comparison of the morphological characteristics between two types of backcross progenies from the common carp and crucian carp. J. N.E. Agric. Univ. 38: 797-800.

Yu X, Zhou T, Li Y, Li K et al. (1989). Chromosomes of Chinese fresh-water fishes [M]. Science Press, Beijing, China.

Yue G, Ho M, Orban L and Komen J (2004). Microsatellites within genes and ESTs of common carp and their applicability in silver crucian carp. Aquaculture 234:85-98.

Zhang Y, Xu P, Lu C, Kuang Y, et al. (2010). Genetic linkage mapping and analysis of muscle fiber-related QTLs in common carp (Cyprinus carpio L.). Mar. Biotechnol. 13: 376-392.

Zheng X, Kuang Y, Zhang X, Lu C, et al. (2011). A genetic linkage map and comparative genome analysis of common carp (Cyprinus carpio L.) using microsatellites and SNPs. Mol. Genet. Genomics 286: 261-277.

Zhou Y and Zheng D (1997). A new calculation method of correlation coefficient test table. Ann. SH Obs. Acad. Sin. 18: 18-23.

Zimdahl H, Nyakatura G, Brandt P, Schulz H, et al. (2004). A SNP map of the rat genome generated from cDNA sequences. Science 303: 807.

Zimmerman AM, Wheeler PA, Ristow SR and Thorgaard GH (2005). Composite interval mapping reveals three QTL associated with pyloric caeca number in rainbow trout, Oncorhynchus mykiss. Aquaculture 247: 85-95. 